\title{
In silico identification of vaccine targets for 2019-nCoV
}

\section{[version 1; peer review: 2 approved]}

\author{
Chloe H. Lee, Hashem Koohy (iD
}

MRC Human Immunology Unit, Medical Research Council (MRC) Human Immunology Unit, MRC Weatherall Institute of Molecular Medicine (WIMM), John Radcliffe Hospital, University of Oxford, Oxford, UK, Oxford, UK

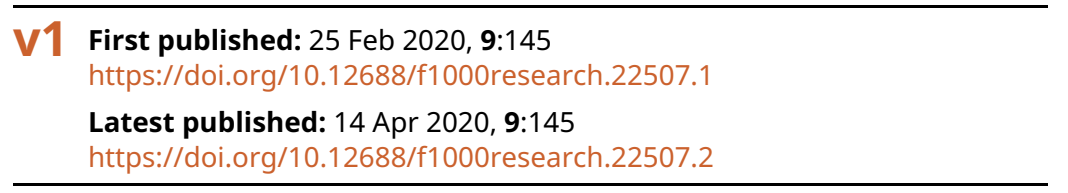

\section{Abstract}

Background: The newly identified coronavirus known as 2019-nCoV has posed a serious global health threat. According to the latest report (18-February-2020), it has infected more than 72,000 people globally and led to deaths of more than 1,016 people in China. Methods: The 2019 novel coronavirus proteome was aligned to a curated database of viral immunogenic peptides. The immunogenicity of detected peptides and their binding potential to HLA alleles was predicted by immunogenicity predictive models and NetMHCpan 4.0. Results: We report in silico identification of a comprehensive list of immunogenic peptides that can be used as potential targets for 2019 novel coronavirus (2019-nCoV) vaccine development. First, we found 28 nCoV peptides identical to Severe acute respiratory syndromerelated coronavirus (SARS CoV) that have previously been characterized immunogenic by T cell assays. Second, we identified 48 $\mathrm{nCoV}$ peptides having a high degree of similarity with immunogenic peptides deposited in The Immune Epitope Database (IEDB). Lastly, we conducted a de novo search of 2019-nCoV 9-mer peptides that i) bind to common HLA alleles in Chinese and European population and ii) have $T$ Cell Receptor (TCR) recognition potential by positional weight matrices and a recently developed immunogenicity algorithm, iPred, and identified in total 63 peptides with a high immunogenicity potential.

Conclusions: Given the limited time and resources to develop vaccine and treatments for 2019-nCoV, our work provides a shortlist of candidates for experimental validation and thus can accelerate development pipeline.

\section{Keywords}

Coronavirus, adaptive immunity, immunogenicity, T cell crossreactivity, vaccine development

\section{Open Peer Review}

Approval Status $\quad 2 \quad 3$

version 2

(revision)

14 Apr 2020

version 1

25 Feb 2020

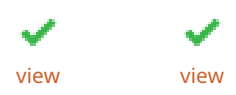

1. Katalin A. Wilkinson (ID), The Francis Crick

Institute, London, UK

Institute of Infectious Disease and Molecular Medicine, University of Cape Town, South

Africa

2. Raffaele De Palma, University of Genova,

Genova, Italy

CNR-Napoli, Naples, Italy

3. Maria Bonsack, German Cancer Research Center (DKFZ), Heidelberg, Germany German Center for Infection Research (DZIF), partner site Heidelberg, Germany

Angelika Riemer (D), German Cancer Research Center (DKFZ), Heidelberg,

Germany

German Center for Infection Research (DZIF), partner site Heidelberg, Germany

Any reports and responses or comments on the 


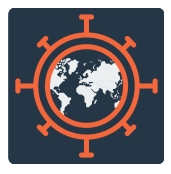

This article is included in the Emerging Diseases

and Outbreaks gateway.

article can be found at the end of the article.

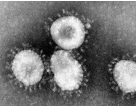

This article is included in the Coronavirus

collection.

Corresponding author: Hashem Koohy (hashem.koohy@rdm.ox.ac.uk)

Author roles: Lee CH: Data Curation, Formal Analysis, Investigation, Methodology, Resources, Software, Validation, Visualization, Writing - Original Draft Preparation, Writing - Review \& Editing; Koohy H: Conceptualization, Data Curation, Funding Acquisition, Investigation, Supervision, Writing - Original Draft Preparation, Writing - Review \& Editing

Competing interests: No competing interests were disclosed.

Grant information: This study was funded by the Medical Research Council Human Immunology Unit.

Copyright: ( $) 2020$ Lee $\mathrm{CH}$ and Koohy H. This is an open access article distributed under the terms of the Creative Commons Attribution License, which permits unrestricted use, distribution, and reproduction in any medium, provided the original work is properly cited.

How to cite this article: Lee $\mathrm{CH}$ and Koohy H. In silico identification of vaccine targets for 2019-nCoV [version 1; peer review: 2 approved] F1000Research 2020, 9:145 https://doi.org/10.12688/f1000research.22507.1

First published: 25 Feb 2020, 9:145 https://doi.org/10.12688/f1000research.22507.1 


\section{Introduction}

The emergence and rapid spread of the recent novel coronavirus known as 2019-nCoV has posed a serious global health threat $^{1}$ and has already caused a huge financial burden ${ }^{2}$. It has further challenged the scientific and industrial community for quick control practices, and equally importantly to develop effective vaccines to prevent its recurrence. In facing a rapid epidemical outbreak to a novel and unknown pathogen, a key bottleneck for a proper and deep investigation, which is fundamental for vaccine development, is the limited -- to almost no -- access of the scientific community to samples from infected subjects. As such, in silico predictions of targets for vaccines are of high importance and can serve as a guidance to medical and experimental experts for the best and timely use of the limited resources.

In this regard, we report our recent effort to computationally identify immunogenic and/or cross-reactive peptides from 2019-nCoV. We provide a detailed screen of candidate peptides based on comparison with immunogenic peptides deposited in the Immune Epitope Database and Analysis Resource (IEDB) database including those derived from Severe acute respiratory syndrome-related coronavirus (SARS CoV) along with de novo prediction from 2019-nCoV 9-mer peptides. Here, we found i) 28 SARS-derived peptides having exact matches in 2019$\mathrm{nCoV}$ proteome previously characterized to be immunogenic by in vitro $\mathrm{T}$ cell assays, ii) $22 \mathrm{nCoV}$ peptides having a high sequence similarity with immunogenic peptides but with a greater predicted immunogenicity score, and iii) $44+19 \mathrm{nCoV}$ peptides predicted to be immunogenic by the iPred algorithm and $1 \mathrm{G} 4$ TCR positional weight matrices respectively.

\section{Results}

Identification of 28 exact matches to SARS CoV

immunogenic peptides by screening all epitopes deposited in IEDB

We collected all peptides in IEDB (3, as of 13-02-2020) reported positive in $\mathrm{T}$ cell assays and have human as the host organism. We then conducted a local sequence alignment of 10 2019-nCoV open reading frames (ORFs) against 35,225 IEDB peptides, and found 28 exact matches. Surprisingly, all identical hits (towards target peptide length $>3$ ) were from SARS-CoV (Table 1, Data Table $1^{4}$ ). These peptides have been shown to bind various HLA alleles, although with higher

Table 1. 28 2019-nCoV peptides having exact matches with immunogenic SARS-CoV peptides.

\begin{tabular}{|c|c|c|c|}
\hline IEDB.peptide & 2019-nCoV.pattern & Antigen.Name & Allele.Name \\
\hline TLACFVLAAV & TLACFVLAAV & Membrane glycoprotein & HLA-A*02:01 \\
\hline AFFGMSRIGMEVTPSGTW & AFFGMSRIGMEVTPSGTW & N protein & \\
\hline AQFAPSASAFFGMSR & AQFAPSASAFFGMSR & nucleocapsid protein & HLA class II \\
\hline AQFAPSASAFFGMSRIGM & AQFAPSASAFFGMSRIGM & $\mathrm{N}$ protein & \\
\hline ILLNKHIDA & ILLNKHIDA & Nucleoprotein & HLA-A*02:01 \\
\hline IRQGTDYKHWPQIAQFA & IRQGTDYKHWPQIAQFA & N protein & \\
\hline KHWPQIAQFAPSASAFF & KHWPQIAQFAPSASAFF & N protein & \\
\hline LALLLLDRL & LALLLLDRL & Nucleoprotein & HLA-A*02:01 \\
\hline RRPQGLPNNTASWFT & RRPQGLPNNTASWFT & nucleocapsid protein & HLA class I \\
\hline YKTFPPTEPKKDKKKK & YKTFPPTEPKKDKKKK & $\mathrm{N}$ protein & \\
\hline ILLNKHID & ILLNKHID & Nucleoprotein & HLA-A*02:01 \\
\hline MEVTPSGTWL & MEVTPSGTWL & nucleocapsid protein & HLA-B*40:01 \\
\hline ALNTLVKQL & ALNTLVKQL & S protein & HLA-A*02:01 \\
\hline FIAGLIAIV & FIAGLIAIV & Spike glycoprotein precursor & HLA-A2 \\
\hline LITGRLQSL & LITGRLQSL & Spike glycoprotein precursor & HLA-A2 \\
\hline NLNESLIDL & NLNESLIDL & S protein & HLA-A*02:01 \\
\hline MAYRFNGIGVTQNVLY & MAYRENGIGVTQNVLY & S protein & HLA-DRB1*04:01 \\
\hline QLIRAAEIRASANLAATK & QLIRAAEIRASANLAATK & S protein & HLA-DRB1*04:01 \\
\hline
\end{tabular}

*SARS-CoV: Severe acute respiratory syndrome coronavirus 
tendency towards HLA-A:02:01, from both class I and class II, and can be target for $\mathrm{CD} 8+$ and $\mathrm{CD} 4+\mathrm{T}$ cells respectively.

\section{Identification of 22 2019-nCoV peptides with high degree} of similarity to previously reported immunogenic viral peptides

In addition to 28 identical hits against SARS CoV, we observed a long tail in distribution of normalized alignment scores between 10 2019-nCoV ORFs and 35,225 IEDB peptides (Figure 1A). We therefore set out to further investigate potential vaccine targets among highly similar sequences.

Taking the normalized alignment score of exact matches as a reference, we extracted 2019-nCoV peptides having score greater or equal to 4. As illustrated in Figure 1A, we observed 45 and 11 peptides having normalized alignment score $\geq 4$ and $\geq 5$ respectively (Figure $1 \mathrm{~A}$ inset). The target peptides were originated from 10 different sources (Figure 1B) where a total 36 peptides were derived from strains associated to SARS CoV. Of interest, we also observed 7 hits having high sequence similarity to targets from Homo sapiens.

In order to investigate the extent to which the difference between the source (2019-nCoV) and target (IEDB) peptides influences the immunogenicity of the source peptides we used a recently published immunogenicity $\operatorname{model}^{5}$ to predict and compare the immunogenicity between the source and target peptides (Data Table $2^{4}$ ).
We could see a similar (close to identical) immunogenicity scores for a number of IEDB and 2019-nCov peptides especially for those with high immunogenicity scores (Figure 2). While all 48 can be potential targets, of particular interest were those having higher immunogenicity score than IEDB peptides. Here, we list 22 out of 48 2019-nCoV peptides that scored higher compared to their targets that have been characterized to be immunogenic (Table 2). In this list 15 (68\%) 2019-nCov peptides have a score higher than 0.5 whereas only $11(50 \%)$ of IEDB get a score immunogenicity score greater than 0.5 .

It is worth noting that in general predicting immunogenicity of given a peptide is challenging and not a fully solved problem, and therefore current models for predicting immunogenicity are suboptimal. iPred is also not an exception. In fact, we could see that a substantial number of IEDB immunogenic peptides were scored $<0.5$ (the threshold score used to classify immunogenic vs non-immunogenic). This led us to ask whether we can gather any other evidence of either immunogenicity or cross-reactivity.

\section{De novo search of immunogenic peptides in 2019-nCoV proteome}

As a complementary reciprocal approach, we conducted a de novo search of immunogenic peptides against the 2019nCov proteome sequence. We scanned 9-mers from 2019-nCoV proteome with a window of 9 amino acids and step length of 1 amino acid (9613 in total). The immunogenicity of 9-mer

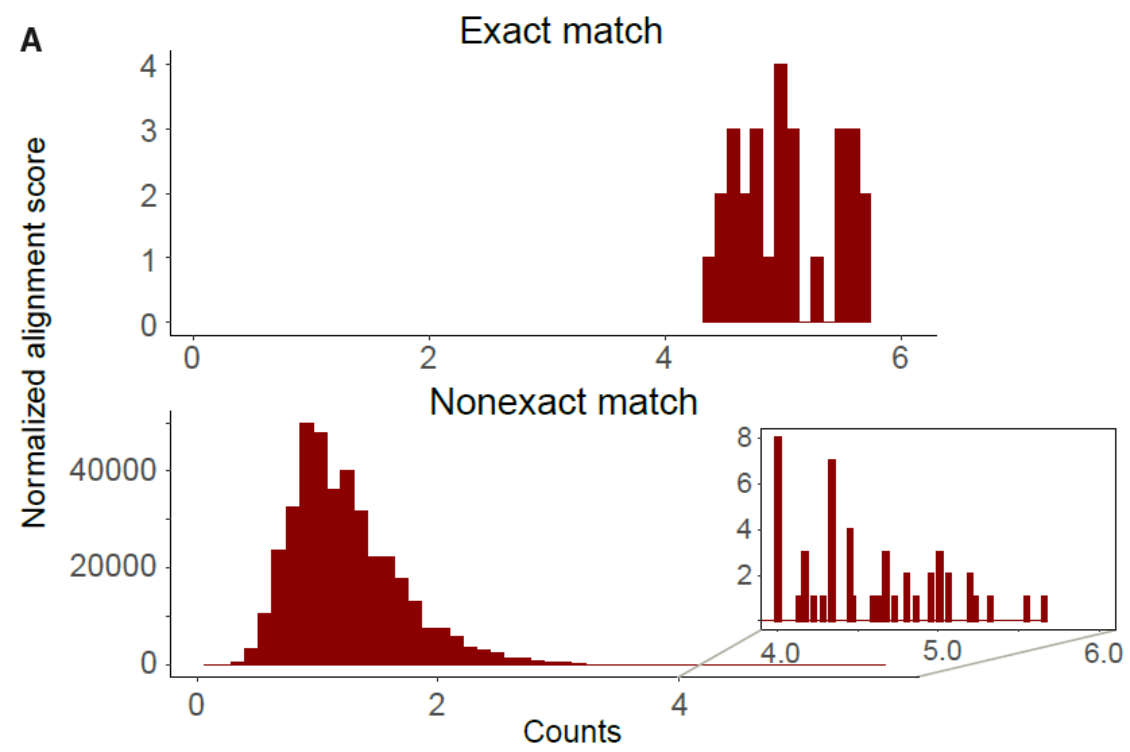

B

\begin{tabular}{|c|c|}
\hline $\begin{array}{c}\text { Source organism of reference } \\
\text { immunogenic peptides }\end{array}$ & Count \\
\hline $\begin{array}{c}\text { Severe acute respiratory } \\
\text { syndrome-related coronavirus }\end{array}$ & 28 \\
\hline Homo sapiens & 7 \\
\hline SARS coronavirus Tor2 & 5 \\
\hline Yellow fever virus 17D & 2 \\
\hline (Unlabelled) & 1 \\
\hline Hepatitis C virus genotype 1 & 1 \\
\hline Human papillomavirus type 16 & 1 \\
\hline SARS coronavirus BJ01 & 1 \\
\hline SARS coronavirus TJF & 1 \\
\hline SARS coronavirus Urbani & 1 \\
\hline Total & 48 \\
\hline
\end{tabular}

Figure 1. 2019-nCoV peptides with high sequence similarity to immunogenic peptides in IEDB. A. Comparison of normalized sequence alignment score for peptides with exact and non-exact matches. B. Number of target peptides grouped by their source organism. 


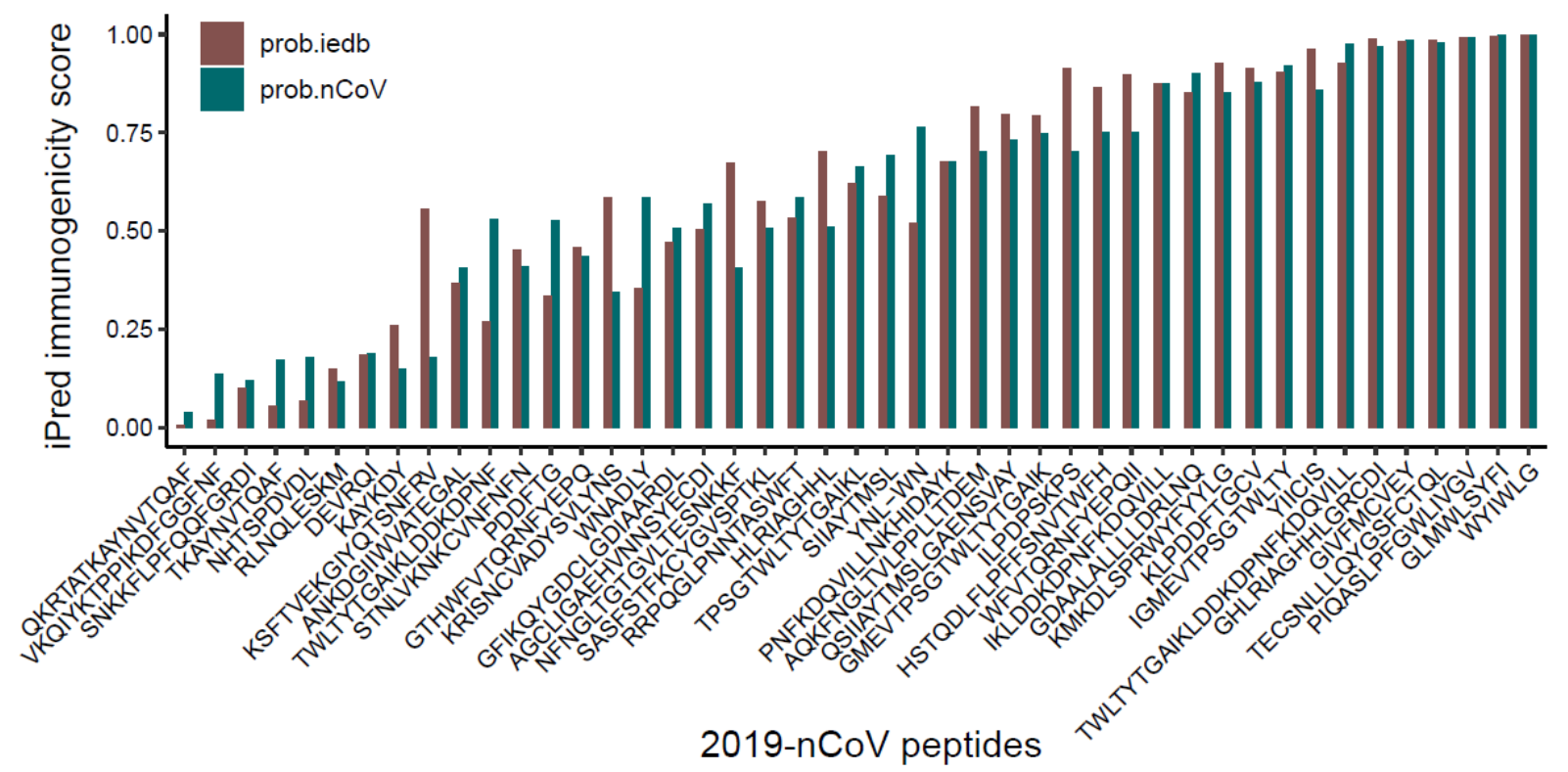

Figure 2. Predicted immunogenicity for IEDB immunogenic vs. 2019-nCoV peptides. 2019-nCoV peptides having a high sequence similarity to immunogenic peptides and their targets were analysed for their immunogenicity potential by iPred algorithm.

Table 2. List of 22 2019-nCoV peptides having a higher predicted immunogenicity score than their target peptides.

\begin{tabular}{|c|c|c|c|}
\hline IEDB.peptide & 2019-nCoV.pattern & IEDB.prob & nCol.prob \\
\hline WYMWLGARY & WYIWLG & 0.999249 & 0.999441 \\
\hline GLMWLSYFV & GLMWLSYFI & 0.995073 & 0.998216 \\
\hline GLVFLCLQY & GIVFMCVEY & 0.98123 & 0.984127 \\
\hline TWLTYHGAIKLDDKDPQFKDNVILL & TWLTYTGAIKLDDKDPNFKDQVILL & 0.925862 & 0.975242 \\
\hline IGMEVTPSGTWLTYH & IGMEVTPSGTWLTY & 0.903518 & 0.919184 \\
\hline GETALALLLLDRLNQ & GDAALALLLLDRLNQ & 0.853114 & 0.900655 \\
\hline TPSGTWLTYHGAIKL & TPSGTWLTYTGAIKL & 0.620894 & 0.662417 \\
\hline SIVAYTMSL & SIIAYTMSL & 0.589694 & 0.693763 \\
\hline RRPQGLPNNIASWFT & RRPQGLPNNTASWFT & 0.533253 & 0.584355 \\
\hline YNLKWN & YNL-WN & 0.520244 & 0.765309 \\
\hline AGCLIGAEHVDTSYECDI & AGCLIGAEHVNNSYECDI & 0.503905 & 0.56813 \\
\hline GFMKQYGECLGDINARDL & GFIKQYGDCLGDIAARDL & 0.471939 & 0.506817 \\
\hline ANKEGIVWVATEGAL & ANKDGI IWVATEGAL & 0.367723 & 0.404796 \\
\hline WNPDDY & WNADLY & 0.355018 & 0.584726 \\
\hline PDDYGG & PDDFTG & 0.334887 & 0.527287 \\
\hline TWLTYHGAIKLDDKDPQF & TWLTYTGAIKLDDKDPNF & 0.27017 & 0.529675 \\
\hline DEVNQI & DEVRQI & 0.18504 & 0.187797 \\
\hline SSKRFQPFQQFGRDV & SNKKFLPFQQFGRDI & 0.098384 & 0.119472 \\
\hline NHDSPDAEL & NHTSPDVDL & 0.067808 & 0.17889 \\
\hline TKQYNVTQAF & TKAYNVTQAF & 0.054818 & 0.171488 \\
\hline VKQMYKTPTLKYFGGFNF & VKQIYKTPPIKDFGGFNF & 0.018685 & 0.135681 \\
\hline QKRTATKQYNVTQAF & QKRTATKAYNVTQAF & 0.004891 & 0.037776 \\
\hline
\end{tabular}


peptides were predicted using iPred and MHC presentation scores were gauged using NetMHCpan $4.0^{6}$ for various HLA types. In this task, we focused on haplotypes common in Chinese and European populations, which include HLA-A*02:01, HLA-A*01:01, HLA-B*07:02, HLA-B*40:01 and HLA-C*07:02 alleles (Data Table $3^{4}$ ).

Based on MHC presentation and immunogenicity prediction, we detected 5 peptides predicted to bind 4 different HLA alleles of which 2 had strong immunogenicity scores (Figure 3). For those 65 strong binders to 3 different HLA types, 39 had immunogenicity scores $\geq 0.5$ (Table 3 ). Collectively this analysis suggests a number of 9-mer immunogenic candidates for further experimental validation.

\section{Immunogenicity of 2019-nCoV peptides to 1G4 CD8+ TCR} molecule

While our de novo candidates are appealing shortlisted targets for experimental validation, it does not provide information about target $\mathrm{T}$ cell receptors (TCRs). We therefore set out to interrogate the possibility of cross reactivity with one well-studied TCR.

$\mathrm{T}$ cell cross-reactivity has been instrumental for the $\mathrm{T}$ cell immunity against both tumor antigens and external pathogens. In that regard, a number of $\mathrm{T}$ cells have been extensively characterized including 1G4 CD8+ TCR, which is known to recognize the 'SLLMWITQC' peptide presented by HLA-A*02:01. We therefore set out to leverage the data from a recently published study ${ }^{7}$ and exploit the possibility of cross reactivity of this TCR to any 2019-nCoV peptide.

Here, we scanned all 9-mers from the 2019-nCoV proteome (9613 peptides) with Binding, Activating and Killing Position Weight Matrices (PWM, see the method section) and associated each peptide with the geometric mean of these three assays as a measure of immunogenicity (Data Table $4^{4}$ ). The distributions of binding, activation and killing scores along with their multiplicative score and geometric mean are illustrated in Figure 4. Based on geometric mean, we observed 20 2019-nCoV peptides with a score $>0.8$ and 516 peptides $>$ 0.7 . The 9-mer peptides with geometric mean $>0.7$ and positive HLA-A*02:01 binding prediction by NetMHCpan 4.0 are listed in Table 4.

We further analysed the MHC binding propensities and gathered peptides not only predicted positive by NetMHCpan but also to have leucine (L) and valine $(\mathrm{V})$ in anchor positions 2 and 9 respectively. This led to identification of 44 2019-nCoV peptides of which 2 peptides had immunogenicity score $>0.7$ and 12 peptides $>0.6$ (Table 5). Thus, here we provide the list of peptides that are potential targets for $1 \mathrm{G} 4 \mathrm{TCR}$ recognition for subjects with HLA-A02:01 haplotype.

\section{Discussion}

In this study we provide a profile of computationally predicted immunogenic peptides from 2019-nCoV for functional

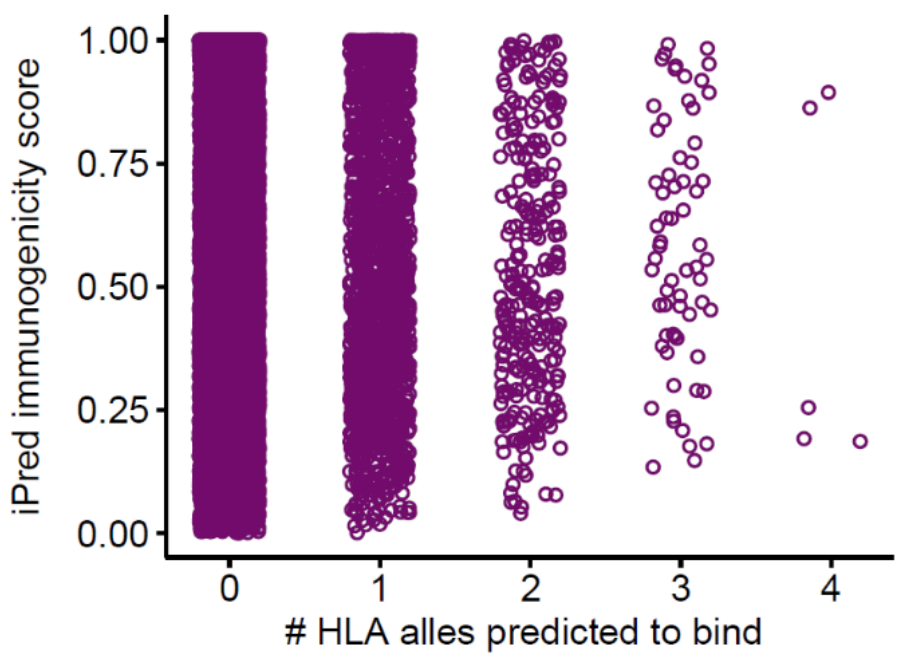

\begin{tabular}{|c|c|c|c|c|c|}
\hline \# of HLA alleles predicted positive & $\mathbf{0}$ & $\mathbf{1}$ & $\mathbf{2}$ & $\mathbf{3}$ & $\mathbf{4}$ \\
\hline \# of 9-mer peptides & 7746 & 1521 & 276 & 65 & 5 \\
\hline P(iPred immunogenicity) $>0.5$ & 3627 & 776 & 134 & 39 & 2 \\
\hline
\end{tabular}

Figure 3. De novo search of 9-mer 2019-nCoV peptides with MHC presentation and immunogenicity potential. The MHC binding was predicted for HLA-A*02:01, HLA-A*01:01, HLA-B*07:02, HLA-B* $40: 01$ and HLA-C*07:02 alleles by NetMHCpan 4.0 and immunogenicity was predicted by iPred. 
Table 3. 2019-nCoV 9-mer peptides predicted to bind 4 different HLA alleles by NetMHCpan 4.0, and those predicted to bind $\geq \mathbf{3}$ HLA alleles and immunogenicity score $\geq \mathbf{0 . 9}$ by iPred. For different alleles, 0 denotes non-binding and 1 denotes binding predicted for specific HLA allele.

\begin{tabular}{|c|c|c|c|c|c|c|c|}
\hline Antigen.epitope & Imm.prob & A0101.NB & A0201.NB & B0702.NB & B4001.NB & C0702.NB & $\begin{array}{c}\text { Total } \\
\text { binding HLA }\end{array}$ \\
\hline VQMAPISAM & 0.893938 & 0 & 1 & 1 & 1 & 1 & 4 \\
\hline AMYTPHTVL & 0.862427 & 0 & 1 & 1 & 1 & 1 & 4 \\
\hline TLDSKTQSL & 0.254998 & 1 & 1 & 1 & 0 & 1 & 4 \\
\hline KVDGVVQQL & 0.191786 & 1 & 1 & 1 & 0 & 1 & 4 \\
\hline KVDGVDVEL & 0.18632 & 1 & 1 & 1 & 0 & 1 & 4 \\
\hline MADQAMTQM & 0.991227 & 1 & 0 & 1 & 0 & 1 & 3 \\
\hline LEAPFLYLY & 0.983072 & 1 & 0 & 0 & 1 & 1 & 3 \\
\hline RTAPHGHVM & 0.972153 & 1 & 0 & 1 & 0 & 1 & 3 \\
\hline IPFAMQMAY & 0.961569 & 1 & 0 & 1 & 0 & 1 & 3 \\
\hline YLQPRTFLL & 0.947743 & 1 & 1 & 0 & 0 & 1 & 3 \\
\hline MMISAGFSL & 0.941318 & 0 & 1 & 1 & 0 & 1 & 3 \\
\hline
\end{tabular}

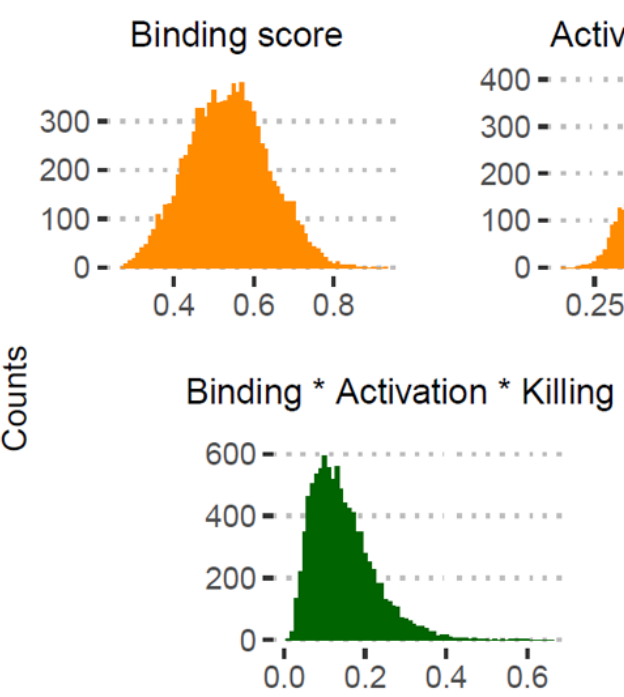

Activation score

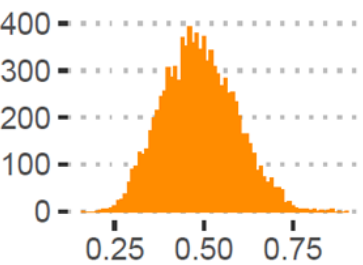

Killing score

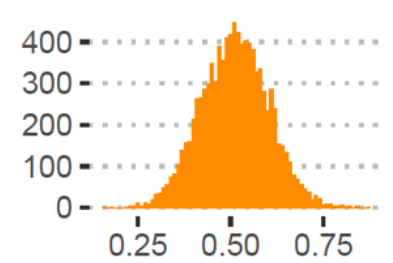

\section{Probability}

Figure 4. Distribution of 1G4 TCR positional weight matrix scores for 2019-nCoV peptides. The positional weight matrices were obtained from 6 and 9613 9-mers generated from 10 2019-nCoV ORFs were computed for their TCR recognition potential.

validation and potential vaccine developments. We are fully aware that an effective vaccine development will require a very thorough investigation of immune correlates to 2019-nCoV. However, due to the emergency and severity of the outbreak as well as the lack of access to samples from infected subjects, such approaches would not serve the urgency. Therefore, computational prediction is instrumental for guiding biologists towards a quick and cost-effective solution to prevent the spread and ultimately help eliminate the infection from the individuals.

With a rising global concern of novel coronavirus outbreak, numerous research groups have started to investigate and 


\begin{abstract}
Table 4. 2019-nCoV 9-mer peptides with geometric mean $\geq 0.7$ by 1 G4 TCR positional weight matrix and predicted positive to bind HLA-A*02:01 by NetMHCpan 4.0 (Rank = NetMHCpan rank)
\end{abstract}

\begin{tabular}{|l|c|c|c|c|c|c|}
\hline Peptide & Binding score & Activation score & Killing score & geoMean & Rank & Binder \\
\hline RIMTWLDMV & 0.866377428 & 0.853995 & 0.776303 & 0.831249 & 0.3481 & SB \\
\hline ALNTLVKQL & 0.802453741 & 0.75073 & 0.785957 & 0.779413 & 0.6159 & WB \\
\hline LLLDRLNQL & 0.809895414 & 0.7752 & 0.741096 & 0.774888 & 0.0423 & SB \\
\hline MIAQYTSAL & 0.766262499 & 0.789511 & 0.749477 & 0.768242 & 0.9238 & WB \\
\hline VLSTFISAA & 0.799672451 & 0.756117 & 0.687278 & 0.746239 & 0.536 & WB \\
\hline NVLAWLYAA & 0.761297552 & 0.686117 & 0.739944 & 0.728423 & 1.4457 & WB \\
\hline RLANECAQV & 0.783161706 & 0.719705 & 0.680504 & 0.726572 & 0.2049 & SB \\
\hline KLLKSIAAT & 0.748896679 & 0.708996 & 0.697463 & 0.718118 & 1.0923 & WB \\
\hline QLSLPVLQV & 0.70128376 & 0.715259 & 0.708405 & 0.708293 & 0.4768 & SB \\
\hline VQMAPISAM & 0.729320768 & 0.698514 & 0.689612 & 0.705612 & 1.4677 & WB \\
\hline LLLTILTSL & 0.7131709 & 0.715194 & 0.680064 & 0.702623 & 0.2712 & SB \\
\hline SVLLFLAFV & 0.736972762 & 0.690855 & 0.679534 & 0.70202 & 1.1449 & WB \\
\hline LMWLIINLV & 0.727847374 & 0.681119 & 0.694007 & 0.700717 & 1.304 & WB \\
\hline
\end{tabular}

Table 5. 2019-nCoV 9-mer peptides having leucine-valine in anchor positions. Peptides have geometric mean $\geq 0.6$ and $\leq 0.7$ (for those $\geq 0.7$, refer to Table 4 ) by 1 G4 TCR positional weight matrix and predicted positive for HLA-A*02:01 binding by NetMHCpan 4.0 (Rank = NetMHCpan rank).

\begin{tabular}{|l|c|c|c|c|c|c|}
\hline Peptide & Binding score & Activation score & Killing score & geoMean & Rank & Binder \\
\hline TLMNVLTLV & 0.723687 & 0.658986 & 0.652178 & 0.677534 & 0.0444 & SB \\
\hline QLEMELTPV & 0.711291 & 0.651003 & 0.608605 & 0.655625 & 1.6769 & WB \\
\hline MLAKALRKV & 0.668756 & 0.610664 & 0.65968 & 0.645854 & 0.3524 & SB \\
\hline GLFKDCSKV & 0.675952 & 0.632375 & 0.594753 & 0.633494 & 0.2677 & SB \\
\hline ALSKGVHFV & 0.652549 & 0.604952 & 0.586236 & 0.613954 & 0.0425 & SB \\
\hline YLNTLTLAV & 0.624147 & 0.610826 & 0.575445 & 0.603119 & 0.0453 & SB \\
\hline
\end{tabular}

publish their findings. At the time of preparing this manuscript, we became aware of a similar study conducted in comparing 2019-nCoV proteome with SARS CoV immunogenic peptides ${ }^{8}$. Our in silico approach takes the search beyond presenting only common immunogenic peptide between SARS and 2019-nCoV and provides the experimental community with a more comprehensive list including de novo and cross reactive candidates. On the other hand, considering the fact that two studies have been accomplished independently with distinct approaches, this serves to demonstrate a high level of confidence in reproducing the results. Reproducibility of computational prediction is always of high importance and becomes even more significant under urgent scenarios as of this outbreak.

Our study also suggests the need for further efforts to develop accurate predictive models and algorithms for the characterization of immunogenic peptides.
In this study, we provide potential immunogenic peptides from 2019-nCoV for vaccine targets that i) have been characterized immunogenic by previous studies on SARS CoV, ii) have high degree of similarity with immunogenic SARS CoV peptides and iii) are predicted immunogenic by combination of NetMHCpan and iPred/1G4 TCR positional weight matrices. Given the limited time and resources, our work serves as a guide to save time and cost for further experimental validation.

\section{Method}

Data acquisition

2019-nCoV open reading frame sequences were downloaded from NCBI (MN908947.3). All sequences subjected for analysis are deposited in GitHub repository.

Data analysis

All subsequent analyses have been conducted in R 3.6.1. 


\section{Sequence similarity comparison}

The sequence similarity between 2019-nCoV open reading frames and previously characterized immunogenic peptides in IEDB was analysed by local alignment using $\mathrm{R}$ 'pairwiseAlignment' function from Biostrings v2.40.2 package. The local alignment utilized BLOSUM62 matrix, gapOpening of 5 and gapExtension of 5 . The alignment score was normalized by length of target peptides.

Immunogenicity prediction

We have used Pred $^{5}$ to predict immunogenicity of each given peptide. Briefly, iPred employs peptides' length and physicochemical properties of amino acids modelled by sums of ten Kidera factors and associates a score to each peptide reflecting its likelihood of recognition by a $\mathrm{T}$ cell.

\section{Predicting presentation by $\mathrm{MHCs}$}

In order to predict peptide binding to MHC we used NetMHCpan V46. This version of NetMHCpan that comes with a number of improvements, incorporate both eluted ligand and peptide binding affinity data into a neural network model to predict MHC presentation of each given peptide.

\section{Predicting cross reactivity to $1 \mathrm{G} 4 \mathrm{TCR}$}

To gauge the level of 1G4 TCR cross-reactivity to list of 2019-nCoV virus, we have leveraged the data from a recently published study ${ }^{7}$. 1G4 or NY-ESO-1-specific TCR is a very well-studied and clinically efficacious TCR which recognize the peptide 'SLLMWITQC' presented by HLA-A*02:01. Karapetyan et al. have recently provided data from three experimental assays reflecting Binding, Activating and Killing upon each mutation at each position of all possible 9-mers using these three datasets. In a similar way to the original paper, we trained three Position Weight Matrices named B, A and $\mathrm{K}$ respectively from Binding, Activating and Killing assay. We defined the cross-reactivity score of a given 9-mer sequence as the geometric mean of $\mathrm{B}, \mathrm{A}$ and $\mathrm{K}$.

We then scanned 2019-nCoV virus protein sequence with each of B, A and K PWMs and associated each of 9613 9-mers with a cross reactivity score. At the same we utilized NetMHCpan and associated each 9-mer with its presentation score. Our final list of cross-reactive candidate peptides were those with a cross-reactivity sore $>=0.8$ and reported as strong binders from NetMHCpan and have ' $\mathrm{L}$ ' and ' $\mathrm{V}$ ' amino acids at anchor positions. The custom $\mathrm{R}$ codes are accessible from GitHub repository (see software availability ${ }^{4}$.

\section{Software availability}

Replication code: https://github.com/ChloeHJ/Vaccine-target-for2019-nCoV.git

Archived source code at time of publication: http://doi.org/ 10.5281/zenodo. $3676908^{4}$

License: Creative Commons Attribution 4.0 International

\section{Data availability}

\section{Source data}

2019-nCoV open reading frame sequences were downloaded from NCBI (MN908947.3).

\section{Underlying data}

Zenodo: In silico identification of vaccine targets for 2019-nCoV (Data tables). http://doi.org/10.5281/zenodo.36768869

This project contains the following underlying data:

- Table1 nCoV peptides having exact match with immunogenic SARS CoV peptides.xlsx (Table of nCoV peptides having exact match with immunogenic SARS CoV peptides)

- Table2 nCoV peptides with high sequence similarity with immunogenic IEDB peptides.csv (Table of peptides with high sequence similarity with immunogenic IEDB peptides)

- Table3 de novo search on 9-mer nCoV for immunogenic peptides by NetMHCpan and iPred.csv (Table of results of de novo search on 9-mer $\mathrm{nCoV}$ for immunogenic peptides by NetMHCpan and iPred)

- Table4 de novo search on 9-mer nCoV for immunogenic peptides by NetMHCpan and PWM.xlsx (Table of results of de novo search on 9-mer $\mathrm{nCoV}$ for immunogenic peptides by NetMHCpan and PWM)

Data are available under the terms of the Creative Commons Attribution 4.0 International license (CC-BY 4.0).

\section{Acknowledgements}

We acknowledge further appreciate assistance and computing support from Unit and WIMM Centre for Computational Biology at MRC Weatherall Institute of Molecular Medicine. We thank G. Napolitani and M. Salio for insightful discussions about the project.
1. World Health Organization: 2019-NCoV Outbreak Is an Emergency of International Concern. World Health Organization, 31 Jan. 2020. Reference Source
2. Kock RA, Karesh WB, Veas F, et al:: 2019-nCoV in context: lessons learned? Lancet Planet Health. 2020; pii: S2542-5196(20)30035-8. PubMed Abstract | Publisher Full Text 
3. Vita R, Mahajan S, Overton JA, et al:: The Immune Epitope Database (IEDB): 2018 update. Nucleic Acids Res. 2019; 47(D1): D339-D343. PubMed Abstract | Publisher Full Text | Free Full Text

4. Lee $\mathrm{CH}$, Koohy $\mathrm{H}$ : In silico identification of vaccine targets for 2019-nCoV (Custom code). Zenodo. 2020.

http://www.doi.org/10.5281/zenodo.3676908

5. Pogorelyy MV, Fedorova AD, McLaren JE, et al.: Exploring the pre-immune landscape of antigen-specific T cells. Genome Med. 2018; 10(1): 68. PubMed Abstract | Publisher Full Text | Free Full Text

6. Jurtz V, Paul S, Andreatta M, et al:: NetMHCpan-4.0: Improved Peptide-MHC Class I Interaction Predictions Integrating Eluted Ligand and Peptide Binding
Affinity Data. J Immunol. 2017; 199(9): 3360-3368.

PubMed Abstract | Publisher Full Text | Free Full Text

7. Karapetyan AR, Chaipan C, Winkelbach $\mathrm{K}$, et al:: TCR Fingerprinting and OffTarget Peptide Identification. Front Immunol. 2019; 10: 2501.

PubMed Abstract | Publisher Full Text | Free Full Text

8. Ahmed SF, Quadeer AA, McKay MR, et al.: Preliminary Identification of Potential Vaccine Targets for the COVID-19 Coronavirus (SARS-CoV-2) Based on SARSCoV Immunological Studies. 2020.

Publisher Full Text

9. Lee $\mathrm{CH}$, Koohy $\mathrm{H}$ : In silico identification of vaccine targets for 2019-nCoV (Data tables). [Data set]. Zenodo. 2020.

http://www.doi.org/10.5281/zenodo.3676886 


\section{Open Peer Review}

\section{Current Peer Review Status:}

\section{Version 1}

Reviewer Report 31 March 2020

https://doi.org/10.5256/f1000research.24839.r60502

(C) 2020 De Palma R. This is an open access peer review report distributed under the terms of the Creative Commons Attribution License, which permits unrestricted use, distribution, and reproduction in any medium, provided the original work is properly cited.

\section{Raffaele De Palma}

1 DIMI, Department of Internal Medicine, University of Genova, Genova, Italy

2 IBBC (Istituto di Biochimica e Biologia Cellulare), CNR-Napoli, Naples, Italy

The Authors use an in silico approach to identify antigenic peptides derived from 2019-nCoV. First, they screened ten open reading frame of 2019-nCoV sequence used the IEDB database, finding a series of peptides potentially immunogenic. The first piece of data relies on the identification of 28 peptides that were exactly matching SARS CoV peptides. Moreover, using combinatory approaches, modelling HLA and TCR binding, they identified 13 peptides potentially able to bind a given TCR in HLA A2 restriction fashion, and cutting a list of peptides able to bind several HLA alleles and characterizing several peptides that may be de novo candidates or crossreactive peptides to be used either to study immune response to 2019-nCoV or to set a vaccine.

Is the work clearly and accurately presented and does it cite the current literature? Yes

Is the study design appropriate and is the work technically sound?

Yes

Are sufficient details of methods and analysis provided to allow replication by others? Yes

If applicable, is the statistical analysis and its interpretation appropriate? Yes

Are all the source data underlying the results available to ensure full reproducibility? Yes

Are the conclusions drawn adequately supported by the results? Yes 
Competing Interests: No competing interests were disclosed.

Reviewer Expertise: T cell responses in Health and Diseases. Modulation of Immune Responses. Clinical Immunology. Vaccines

\section{I confirm that I have read this submission and believe that I have an appropriate level of expertise to confirm that it is of an acceptable scientific standard.}

Reviewer Report 05 March 2020

https://doi.org/10.5256/f1000research.24839.r60504

(C) 2020 Wilkinson K. This is an open access peer review report distributed under the terms of the Creative Commons Attribution License, which permits unrestricted use, distribution, and reproduction in any medium, provided the original work is properly cited.

\section{Katalin A. Wilkinson}

1 Tuberculosis Laboratory, The Francis Crick Institute, London, UK

2 Institute of Infectious Disease and Molecular Medicine, University of Cape Town, South Africa

This is an important manuscript identifying potential vaccine targets for 2019-nCoV, using computational prediction. In the absence of patient samples, such approaches are instrumental to guide towards quick and efficient identification of vaccine candidates. The authors used 10 open reading frame sequences of 2019-nCoV deposited at NCBI and conducted sequence alignment against immunogenic peptides deposited in the Immune Epitope Database and Analysis Resource (IEDB) database. They identified 28 peptides with sequences matching exactly to severe acute respiratory syndrome-related coronavirus (SARS CoV), that have previously been characterised as immunogenic by $T$ cell assays. These findings are very promising and have the added benefit of potentially developing a vaccine against both SARS and COVID-19.

Additional peptides were identified to most likely bind common Chinese and European HLA alleles and have high immunogenicity potential. The authors provide a shortlist of peptides as potential vaccine candidates. While this manuscript presents a good model for identifying such targets, a comment should be included in the discussion about the necessity of expanding the analysis to include wider HLA allele types, considering that the virus is likely to spread worldwide.

Minor comment: Please explain the methods and analysis in greater details, including the following terms:

1. 'Towards target peptide length $>3$ '.

2. 'Normalized alignment scores' (and their scale, such as the significance of 4 for the data presented in Figure 1).

3. The importance of leucine (L) and valine (V) in anchor positions for MHC binding (for data presented in Table 5).

Is the work clearly and accurately presented and does it cite the current literature? 
Yes

Is the study design appropriate and is the work technically sound?

Yes

Are sufficient details of methods and analysis provided to allow replication by others? Partly

If applicable, is the statistical analysis and its interpretation appropriate?

Not applicable

Are all the source data underlying the results available to ensure full reproducibility? Yes

Are the conclusions drawn adequately supported by the results?

Yes

Competing Interests: No competing interests were disclosed.

Reviewer Expertise: T cell immunology

I confirm that I have read this submission and believe that I have an appropriate level of expertise to confirm that it is of an acceptable scientific standard.

Author Response 07 Apr 2020

Hashem Koohy, Medical Research Council (MRC) Human Immunology Unit, MRC Weatherall Institute of Molecular Medicine (WIMM), John Radcliffe Hospital, University of Oxford,

Oxford, UK, Oxford, UK

Dear Prof Wilkinson,

Thank you so much for your time and valuable suggestions. In our new and revised version we have taken your suggestions into consideration.

Best regards

Hashem Koohy

Competing Interests: No competing interests were disclosed. 
The benefits of publishing with F1000Research:

- Your article is published within days, with no editorial bias

- You can publish traditional articles, null/negative results, case reports, data notes and more

- The peer review process is transparent and collaborative

- Your article is indexed in PubMed after passing peer review

- Dedicated customer support at every stage

For pre-submission enquiries, contact research@f1000.com 